\title{
Cohort Profile: Swedish Twin Study on Prediction and Prevention of Asthma (STOPPA)
}

\author{
Catarina Almqvist, ${ }^{1,2}$ Anne K. Örtqvist, ${ }^{1}$ Vilhelmina Ullemar, ${ }^{1}$ Cecilia Lundholm, ${ }^{1}$ Paul Lichtenstein, ${ }^{1}$ and \\ Patrik K. E. Magnusson ${ }^{1}$ \\ ${ }^{1}$ Department of Medical Epidemiology and Biostatistics, Karolinska Institutet, Stockholm, Sweden \\ ${ }^{2}$ Astrid Lindgren Children's Hospital, Lung and Allergy Unit, Karolinska University Hospital, Stockholm, Sweden
}

\begin{abstract}
Asthma is a common childhood disease and several risk factors have been identified; however, the impact of genes and environment is not fully understood. The aim of the Swedish Twin study On Prediction and Prevention of Asthma (STOPPA) is to identify environmental (birth characteristics and early life) and genetic (including epigenetic) factors as determinants for asthmatic disease. Based on the Child and Adolescent Twin Study in Sweden (CATSS) (parental interview at 9 or 12 years, $N \sim 23,900$ ) and an asthma and/or wheezing algorithm, we identified a sample of monozygotic (MZ) and dizygotic (DZ) same-sexed twin pairs. The twin pairs were classified as asthma concordant (ACC), asthma discordant (ADC) and healthy concordant (HCC). A sample of 9- to 14-year-old twins and their parents were invited to participate in a clinical examination. Background characteristics were collected in questionnaires and obtained from the National Health Registers. A clinical examination was performed to test lung function and capacity (spirometry with reversibility test and exhaled nitric oxide) and collect blood (serology and DNA), urine (metabolites), feces (microbiota), and saliva (cortisol). In total, 376 twin pairs (752 individual twins) completed the study, response rate $52 \%$. All participating twins answered the questionnaire and $>90 \%$ participated in lung function testing, blood-, and saliva sampling. This article describes the design, recruitment, data collection, measures, and background characteristics, as well as ongoing and planned analyses in STOPPA. Potential gains of the study include the identification of biomarkers, the emergence of candidates for drug development, and new leads for prevention of asthma and allergic disease.
\end{abstract}

Keywords: asthma, allergic disease, concordance, discordance, epidemiology, epigenetic, genetic, lung function, twin

Asthma is an inflammatory and obstructive disease of the airways, with increasing prevalence around the world (Asher et al., 2006). Heritability estimates specific to populations and given environments have indicated genetic factors to be responsible for $60-80 \%$ of liability to asthma and respiratory measures, and the remaining 20-40\% due to environmental influence (Lichtenstein \& Svartengren, 1997; Los et al., 2001; Wu et al., 2010). There has been an upsurge in observational birth cohort studies and randomized controlled trials, along with large consortia, to better understand the impact of environmental risk factors of asthma and allergic disease (Custovic \& Simpson, 2004; Lau et al., 2002; Mihrshahi et al., 2001; Wickman et al., 2002; Wijga et al., 2001). Several risk factors have been identified, but there are still controversies over many exposures.

We have recently shown that there is an association between birth weight and subsequent asthma (Ortqvist et al., 2009) and eczema (Lundholm et al., 2010) in twins. The observed association was independent of gestational age, familial environment and genetic factors, implying that altered fetal growth affects early metabolic, immunologic, or physiologic mechanisms in utero, leading to subsequent disease. The fetal origins hypothesis (Barker, 1995) states that the fetal malnutrition in utero may result in disproportionate neonatal growth that may lead to catch-up growth, causing an increased risk of subsequent obesity (Stettler et al., 2002). Both asthma and obesity seem to have their commencement in early childhood (Drever et al., 2010; Snethen et al., 2007). It is possible that other unshared environmental exposures may predispose individuals to both these conditions and may explain how they are associated.

RECEIVED 26 November 2014; ACCEPTED 25 February 2015. First published online 22 April 2015.

ADDRESS FOR CORRESPONDENCE: Catarina Almqvist, Professor MD, Department of Medical Epidemiology and Biostatistics, Box 281, Karolinska Institutet, SE-171 77 Stockholm, Sweden. E-mail: catarina.almqvist@ki.se 
However, most exposures are influenced by genetic effects, and therefore genetic confounding cannot be excluded. This is one area where twin studies may provide an alternative and very powerful approach.

There is also accumulating epidemiologic (BraunFahrlander et al., 1999), clinical (Matricardi, 2002), and experimental (Blaser et al., 2008) data supporting the 'hygiene hypothesis' (Strachan, 1989), and it would not be far-fetched to postulate that perturbations in the gastrointestinal microflora could disrupt the normal mechanisms of immunological tolerance in the mucosa (Noverr \& Huffnagle, 2005), leading to an increased risk of several childhoodonset allergic disorders. There is also an association between early measures of stress and asthma (Fang et al., 2011) and although findings are inconsistent (Vink et al., 2013), measures of stress in saliva cortisol may affect allergic symptoms (Stenius et al., 2011). Thus, if the effects are causal, it is expected that the associations between objective measures such as spirometry, serology, gut microflora, as well as saliva cortisol and parent-reported asthma, remain also within twin pairs.

Twin siblings share genes (100\% if MZ and on average $50 \%$ of their segregating genes if $\mathrm{DZ}$ ), some intrauterine exposures, maternal factors and early environment. These renowned differences in genetic similarity, together with the assumption that twins within a pair share environmental exposures to the same degree irrespective of zygosity, create the foundation for the twin design to explore the effects of genetic and environmental variance on a phenotype. Differences between MZ twins may either be an effect of purely environmental factors or genetic variation that arises after the fragmentation of the fertilized embryo, and MZ twin pairs discordant for asthma are especially powerful to study the effect of early environment on disease development. It has been demonstrated that genetic variation can occur after birth, either through somatic mutations or epigenetic changes (Martino et al., 2013; Wong et al., 2010). It has also been hypothesized that epigenetics may be a molecular link between environmental factors and phenotypic changes (Zhang et al., 2012). A few epigenetic mechanisms have been proposed to be involved in the development of asthma (Hollingsworth et al., 2008; Liu et al., 2008). However, more direct mechanistic links to asthma pathogenesis need to be determined.

The overarching purpose of this research program is to identify environmental (birth characteristics and early life), genetic and epigenetic factors as determinants for asthmatic disease. We will use the powerful approach of questionnaires and objective measures based on clinical examinations in discordant and concordant $\mathrm{MZ}$ and DZ twins.

\section{Material and Methods}

\section{Study Design and Population}

In 2004, the Swedish Twin Registry initiated the CATSS, including all twins born from July 1992 onwards. The twins were identified from the Swedish Medical Birth Register and the parents answered a telephone interview on birth characteristics, zygosity, and parental background factors, as well as outcomes such as asthma and wheeze at 9 or 12 years of age $(N \sim 23,900$ twins in March 2014, response rate 79\%) (Anckarsater et al., 2011; Magnusson et al., 2013). The questions on zygosity have been validated with DNA markers in previous studies and proved correct in 95\% (Lichtenstein et al., 2002).

Based on the CATSS interview ascertainment, the STOPPA was initiated in May 2011. Validated questions on asthma ever (yes/no) and wheezing (current or after 3 years of age/no) from the International Study of Asthma and Allergies in Childhood, ISAAC (Asher et al., 1995) were used to create an algorithm to identify twins discordant and concordant for asthma (Figure 1). Three groups of MZ and DZ same-sexed twin pairs were identified: (1) both twins with asthma or wheezing (ACC), (2) one twin with asthma or wheezing and the other without (ADC), and (3) none of the twins with asthma or wheezing (HCC).

From each group (ACC, ADC, and HCC), 9- to 14-yearold twin pairs and their parents were invited to participate in a clinical examination including questionnaires and objective measures. Twins already recruited to other CATSS substudies were excluded ( $n=10$ pairs). Eligible twins were invited in rounds related to examination site, date and age groups, to obtain equal sizes of the subgroups. The HCC was the largest group, so in order to obtain an equal size of the groups, we invited a larger proportion of the ACC and ADC groups. The families were contacted through an invitation letter, followed by a telephone call to inform them about the study. A clinical examination including questionnaires and objective measures were performed at Karolinska Institutet (Stockholm), Queen Silvia Children's Hospital (Göteborg), Linköping's University hospital, Centralsjukhuset in Karlstad, Norrland's University hospital (Umeå), Lund Children's Hospital, Lomma Primary Health Care Centre, and Centrallasarettet in Växjö. The protocol between the pilot and the full scale study were identical apart from minor revisions of the questionnaire and collection of urinary samples during the last year of the study. After a successful pilot study that included $15 \mathrm{MZ}$ twin pairs in Stockholm and Uppsala, the full-scale study started in November 2011 and was finalized in June 2014.

\section{Questionnaires}

At the test center, the parents were invited to answer a questionnaire divided into eight main themes: parental background; lifestyle and medical history, along with questions on each twin's lifestyle; general health status; medical history; puberty; and home environment. The questionnaire was designed to collect information on factors that may be associated with childhood asthma, allergies and comorbidities, along with previously validated questions. Questions on twins' medical history included respiratory diagnoses in 


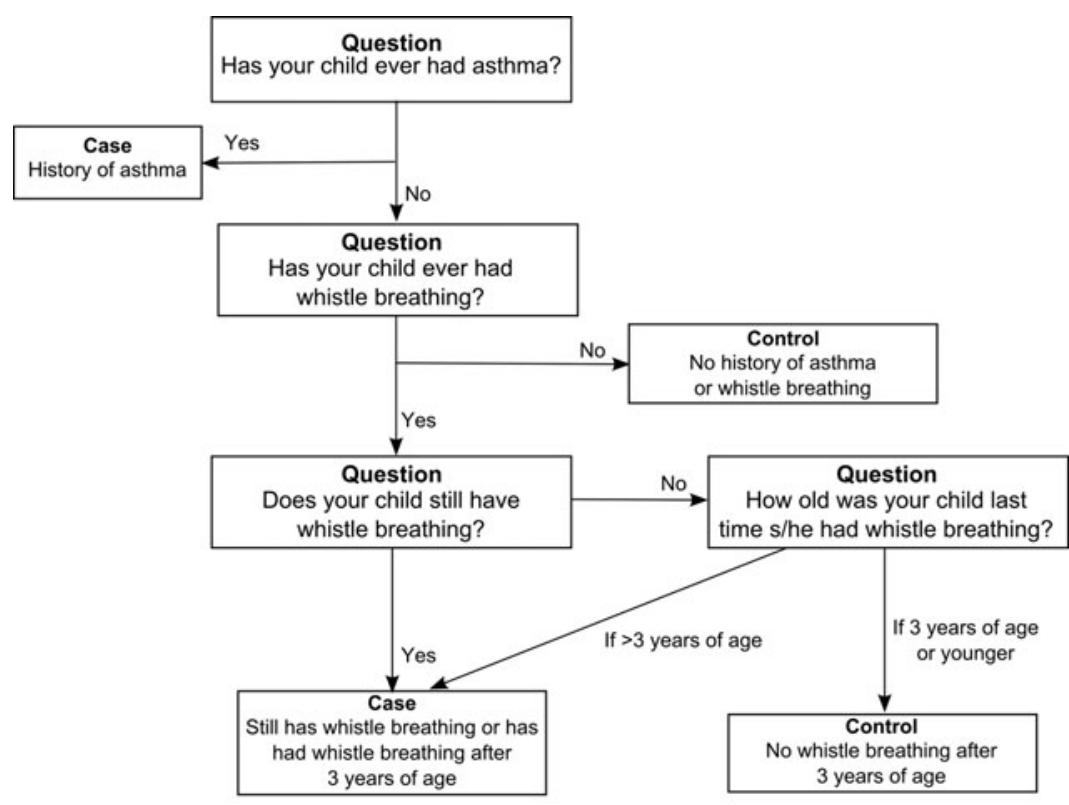

FIGURE 1

Algorithm to identify twins discordant and concordant for asthma, based on validated questions on asthma and wheezing from the International Study of Asthma and Allergies in Childhood (ISAAC).

infancy, wheezing and asthma, including doctor's diagnosis, age at onset and frequency (Asher et al., 1995; Danell et al., 2013; Ekstrom et al., 2014; Wickman et al., 2002). For lifestyle, general health and home environment, established questionnaires were used (Almqvist et al., 2011; Anckarsater et al., 2011).

Each twin was invited to answer a questionnaire that was divided into four themes: lifestyle, medical history, puberty, and stress. Questions on puberty were those in the Pubertal Development Scale developed and validated by Petersen et al. (1988). The Perceived Stress Scale was used for the pilot (Cohen et al., 2011) and replaced by the 21-item questionnaire 'Stress in Children' (SiC), which has been validated against Beck Youth Inventory scales and objective measures of stress (Osika et al., 2007), in the full-scale study.

The Asthma Control Test (ACT), a trademark of QualityMetric Incorporated, including five items on symptoms and medication during the last 4 weeks, was used for twins $>12$ years of age and the Childhood Asthma Control Test (C-ACT) answered by twins $<12$ years (four items) and parents (three items), with permission from GlaxoSmithKline (Liu et al., 2010; Nathan et al., 2004).

The parental and twin questionnaires were slightly revised between the pilot phase and full-scale study and a few questions on early life respiratory diagnoses and exposure to antibiotics, physical activity, pet exposure, and stress were added or modified. A research nurse contacted and completed missing information for the majority of pilot participants in retrospect.

\section{Clinical Examination}

Spirometry. Lung function tests to measure how an individual inhales or exhales volumes (liters) of air as a function of time (seconds), was carried out with SpiroStar (Pro version, Medikro, Kuopio, Finland) in the majority of study participants. Approximately 130 twins were tested using the Jaeger MasterScreen-IOS system (Carefusion Technologies, San Diego, California) at one of the test centers in Stockholm. The procedures were performed in accordance with American Thoracic Society/European Respiratory Society recommendations (Miller et al., 2005). Both systems were calibrated daily using a $3 \mathrm{~L}$ syringe. A reversibility test was included and the procedure was repeated at least three times before and $15 \mathrm{~min}$ after inhalation of terbutalin $0.5 \mathrm{mg}$.

Fractional exhaled NO (FeNO). Levels of FeNO, a noninvasive marker of airway inflammation (Dweik et al., 2011) were measured with a hand-held electrochemical analyzer (NIOX Mino, Aerocrine, Solna, Sweden) with an expiration time of $10 \mathrm{~s}$ in the majority of study participants. Approximately 100 twins were tested with the CLD 88 FeNO test analyzer (Ecomedics, Duernten, Switzerland) at one of the test centers in Stockholm. Each subject performed the test at least twice; if there was $>5 \%$ difference between the first two measurements a third attempt was performed.

\section{Biosamples}

Blood. At the test centers EDTA whole blood for analyses of DNA (4 mL), complete blood count (CBC; 
$4 \mathrm{~mL}$ ) and plasma aliquots (from EDTA $10 \mathrm{~mL}$ ) were collected, along with tubes of lithium heparin with gel for analyses of proteins and PAXgene Blood RNA Tube (PreAnalytiX GmbH, Hombrechtikon, Switzerland) for analyses of RNA.

Gut microflora. To investigate differences in the twins' gut microflora, fecal samples were taken at home and brought to the test center. The fecal samples were collected in a container with screw cap and spoon (length $101 \mathrm{~mm} / \varnothing$ $16.5 \mathrm{~mm}$ ), and the twins were asked to fill at least twothirds of the container. The samples were frozen at home in $-18{ }^{\circ} \mathrm{C}$ in a pre-frozen cool transport container. The container with the samples was transported to the test center in Styrofoam casing to ensure that the samples remained frozen.

Saliva. Samples of morning and night saliva for analysis of cortisol levels, a marker of stress, were collected at home in a commercial Salivette tube containing a cotton wool swab. The swab was rotated in the mouth for at least 1 min and then inserted back into the tube and kept in $+5^{\circ} \mathrm{C}$ before transportation to the test center. At the test center the cortisol samples were centrifuged and thereafter primarily stored at $-20^{\circ} \mathrm{C}$.

Urinary metabolites. Urinary samples were collected at two time points at the test center; on arrival and before leaving the center. The subjects were asked to pass all urine directly into a measuring container and the total volume at each collection was noted. The urine was thereafter transferred to five $8 \mathrm{~mL}$ test tubes with caps before primary storage at $-20^{\circ} \mathrm{C}$.

All test tubes and material used to collect fecal samples, saliva and urinary samples were provided from SARSTEDT AG \& Co, Nümbrecht, Germany. At the end of each data collection, all samples, except those that were analyzed directly at the test site for the full scale study (CBC), were transported to and stored at Karolinska Institutet's Biobank at $-80{ }^{\circ} \mathrm{C}$, until analyzed for its specific cause.

\section{National Registers}

The universal use of the Personal Identity Number, a unique identifier for each resident in Sweden, enables unambiguous linkage between the registers held by the National Board of Health and Welfare and the Swedish Twin Registry. Information on pre- and perinatal factors such as birth weight, birth length, gestational age, head circumference, and mode of delivery from the Medical Birth Register can be linked to each twin. Information on the twins' asthma medications from the Swedish Prescribed Drug Register and diagnoses in the National Patient Register provides additional information on asthma status. We have recently shown that asthma medication is a suitable proxy for asthmatic disease in children in this age group (Ortqvist et al., 2013).

\section{Child and School Health Records}

To investigate how growth over time affects the risk of subsequent asthma, consent to collect the twins' child and school health records was collected.

\section{Statistical Analyses}

For future studies, and dependent on the specific research question, the collected data from questionnaires, objective measures, and samples will be analyzed with twin design using structural equation models (SEM) or co-twin control analyses where discordant twin pairs are regarded as matched case-control pairs. Twin pairs who are discordant for the outcome will be analyzed with the non-affected co-twin as a matched control to the affected twin, with the exposure being the individual background characteristic. The outcome-concordant twins and HCC twins will be used as control groups. Analyses will be weighted, in order to account for the difference in sampling fractions in the six sampling groups (MZ ACC, MZ ADC, MZ HCC, DZ ACC, DZ ADC, and DZ HCC).

Descriptive statistics on numbers of examined twins, collected information from questionnaire, and clinical measures presented here were calculated by Stata statistical software, version 13 .

The authors assert that all the procedures contributing to this work comply with the ethical standards of the relevant national and institutional committees on human experimentation and with the Helsinki Declaration of 1975, as revised in 2008. The study was approved by the regional ethical review board in Stockholm, Sweden. Informed consent for the study was obtained from all the participants and their parents.

\section{Results}

In total, 6,174 twins were eligible, 1,448 were contacted, 870 agreed to participate and 752 came to the clinical examination, a response rate of 52\% (Figure 2). During recruitment, our aim was to recruit as many ADC as possible. Among those eligible, the proportion of HCC twin pairs (65\%) was higher than ADC (22\%) and ACC (13\%), and the proportion of $\mathrm{MZ}$ twin pairs was lower in the ADC $(27 \%)$ than the ACC group (55\%). In the final study population $38 \%$ of the twin pairs were ADC (49\% MZ), 31\% ACC (61\% MZ), and 31\% HCC (55\% MZ). Altogether, 410 twins (205 twin pairs) were MZ (54\%) and 342 (171 pairs) DZ.

The proportion of males (53\%) was slightly higher than females in the final study population. The majority of twins were born 1999-2000 (46\%) or 2001-2002 (34\%) and had their clinical examination in Stockholm $(60 \%)$, Göteborg (19\%), or Lund/Lomma (8\%) — see Table 1. The 
TABLE 1

Study Population Characteristics

\begin{tabular}{|c|c|c|c|c|c|c|c|}
\hline & \multirow[b]{2}{*}{$N(\%)$} & \multicolumn{3}{|c|}{ Monozygotic, $N=410$} & \multicolumn{3}{|c|}{ Dizygotic, $N=342$} \\
\hline & & $\mathrm{ACC}$ & ADC & $\mathrm{HCC}$ & $A C C$ & $A D C$ & $\mathrm{HCC}$ \\
\hline Total & $752(100)$ & $142(35 \%)$ & $140(34 \%)$ & $128(31 \%)$ & $92(27)$ & $146(43)$ & $104(30)$ \\
\hline \multicolumn{8}{|l|}{ Sex } \\
\hline Male & $396(53)$ & $92(65)$ & $66(47)$ & $58(45)$ & $56(61)$ & $78(53)$ & $46(44)$ \\
\hline Female & $356(47)$ & $50(35)$ & $74(53)$ & $70(55)$ & $36(39)$ & $68(47)$ & $58(56)$ \\
\hline \multicolumn{8}{|l|}{ Birth year } \\
\hline 1997-1998 & $132(18)$ & $36(25)$ & $28(20)$ & $40(31)$ & $8(9)$ & $16(11)$ & $4(4)$ \\
\hline 1999-2000 & $344(46)$ & $52(37)$ & $44(31)$ & $66(52)$ & $32(35)$ & $84(58)$ & $66(63)$ \\
\hline 2001-2002 & $256(34)$ & $50(35)$ & $62(44)$ & $22(17)$ & $46(50)$ & $44(30)$ & $32(31)$ \\
\hline 2003-2004 & $20(3)$ & $4(3)$ & $6(4)$ & $0(0)$ & $6(7)$ & $2(1)$ & $2(2)$ \\
\hline \multicolumn{8}{|l|}{ Study place } \\
\hline Stockholm & $450(60)$ & $78(55)$ & $78(56)$ & $66(52)$ & $60(65)$ & $118(81)$ & $50(48)$ \\
\hline Gothenburg & 140 (19) & $38(27)$ & $38(27)$ & $40(31)$ & $6(7)$ & $2(1)$ & $16(15)$ \\
\hline Linköping & $28(4)$ & $8(6)$ & $4(3)$ & $6(5)$ & $2(2)$ & $8(5)$ & $0(0)$ \\
\hline Karlstad & $20(3)$ & $4(3)$ & $0(-)$ & $0(0)$ & $0(0)$ & $12(8)$ & $4(4)$ \\
\hline Umeå & $22(3)$ & $6(4)$ & $0(-)$ & $10(8)$ & $4(4)$ & $0(0)$ & $2(2)$ \\
\hline Lund (incl. Lomma) & $60(8)$ & $8(6)$ & $14(10)$ & $6(5)$ & $12(13)$ & $6(4)$ & $14(14)$ \\
\hline Växjö & $32(4)$ & $0(-)$ & $6(4)$ & $0(0)$ & $8(9)$ & $0(0)$ & $18(17)$ \\
\hline
\end{tabular}

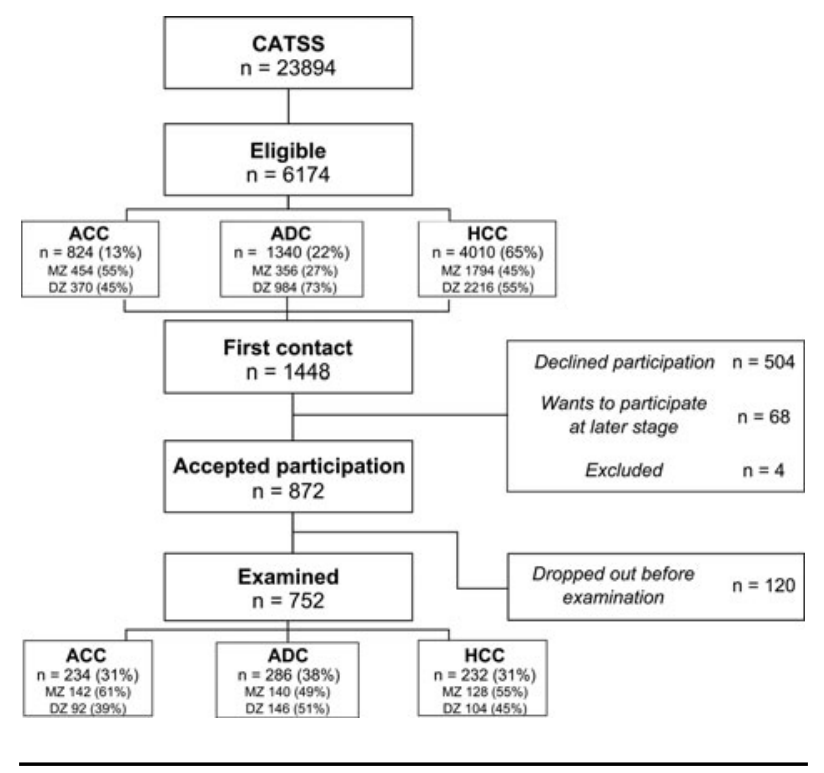

FIGURE 2

Flowchart on recruitment and final study population. Asthma concordant (ACC), asthma discordant (ADC) and healthy concordant (HCC) twin pairs.

distribution of males and females, year of birth, and site for clinical examination for the separate MZ and DZ subgroups was similar to the total study population, except that the proportion of males was slightly lower in MZ ADC (47\%), MZ HCC (45\%), and DZ HCC (44\%).

In the final sample, all twins and their parents answered the questionnaires, $100 \%$ of the twins participated in measures of height and weight, and the majority of twins took part in lung function measures (spirometry 98\%, FeNO 94\%), blood sampling (>90\%), and saliva (97\%) - see Figure 3. Fecal samples were collected in $47 \%$ and urinary samples in $19 \%$.

\section{Discussion}

This is the first twin study on asthma concordant, asthma discordant and healthy concordant twin children examined with questionnaires and a thorough clinical examination. The study design has a powerful approach, with the invitation of a large sample of 9- to 14-year-old Swedish $\mathrm{MZ}$ and DZ twins based on the total population. There are a few previous similar studies on adult populations but most of them were a smaller size (Lund et al., 2007; Wu et al., 2010). This article describes the design, recruitment, data collection, measures, background characteristics as well as ongoing and planned analyses in STOPPA.

We collected questionnaires and objective data from lung function tests and biological samples. Well-validated ISAAC questions on asthma and allergy, including queries on current asthma, were incorporated into the questionnaires to update information from the CATSS questionnaire, which defined the asthma dis- and concordant groups ACC, ADC, and HCC. We also obtained information on asthma severity from the ACT survey, and additional phenotypic information will be obtained from lung functions tests and exhaled NO. In addition, we will have the possibility to link data to the national health registers (diagnoses and prescribed drugs) for further phenotypic information.

Questionnaires were completed by both parents and twins at the clinical test center, which ensured a high response rate. The majority of children also participated in lung function tests and consented to biosamples being taken. We were able to travel to several sites and thus ensure that twins throughout Sweden were examined in a standardized way, using mostly identical equipment and the same team of study nurses. There were also some inherent limitations in the study. Twins were examined throughout the year, including during pollen seasons and autumn, 


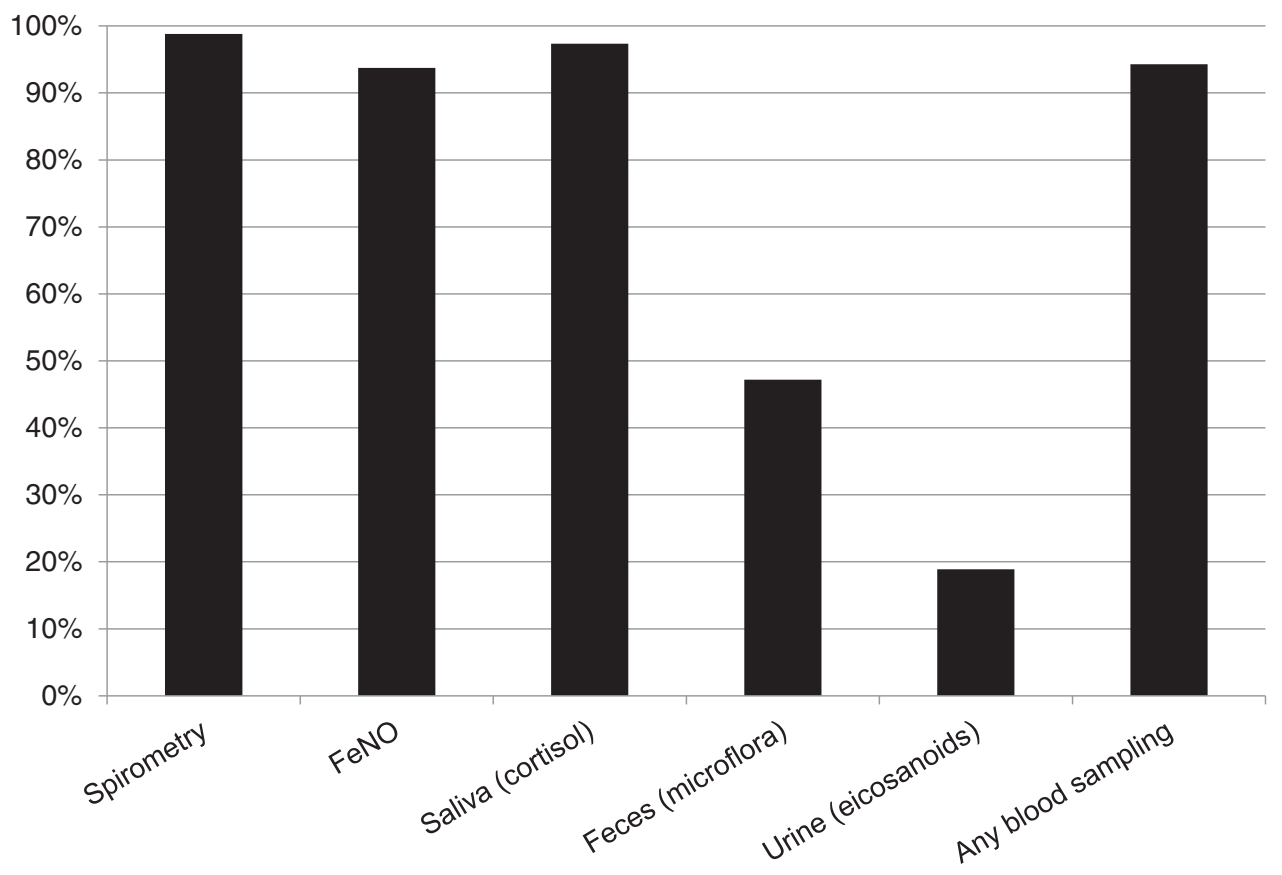

FIGURE 3

Proportion of study participants that participated in lung function testing (spirometry and FeNO), saliva, feces and urinary sampling as well as blood sampling.

when there is a higher risk of upper respiratory tract infections, which may have affected some of the responses. Also, although we aimed to invite all ADC and ACC twins, we had to perform examinations related to site, date, and age group and thus made a great effort to obtain equal sizes of the subgroups and a response rate of $>50 \%$.

MZ twins discordant for asthma have interested clinicians and researchers over the years. Following developments in biotechnology, with the new possibilities to perform systematic large-scale investigations of DNA sequencing, DNA modifications, RNA, metabolites and proteins levels, the interest in using twins for molecular epidemiology has increased, the rationale being that identified molecular differences may reflect causal mechanisms involving environmental exposures and/or constitute a direct cause of the phenotypic difference. DZ twins also give additional information. Mutations occurring after the split of the initial intact embryo and epigenetic (DNA methylation, histone acetylation) differences have been put forward as promising candidate mechanisms.

This article describes the design, recruitment, data collection, measures, background characteristics as well as ongoing and planned analyses in STOPPA. We will make comparisons on the effect of environmental factors on outcomes across discordant and concordant twin pairs and initiate novel analyses on genome-wide methylation in extracted DNA. Potential gains of the study include the identification of biomarkers, the emergence of candidates for drug development, translational modeling, and new leads for prevention of asthma and allergic disease.

\section{Acknowledgments}

First, we direct our greatest appreciation to the twins and parents of the STOPPA cohort, without whose participation this study could not have been performed. We are also indebted to the STOPPA research nurses Karin Dellenvall, Malena Kjellén, Jeanette Tedborn, Monika Nordenbrand, Faiza Abdillahi, Eva Thunberg, Fanny Ståhl and Mikaela Engdahl for their excellent data collection, and to Gerd Agerberg, Michael Broms, Camilla Palm and Rozita Broumandi for superb data managing. Harald Haapala is greatly acknowledged for his contribution to the project as a patient research partner.

We also want to direct our thanks to the Queen Silvia Children's Hospital (Göteborg), Linköping's University Hospital, Centralsjukhuset in Karlstad, Norrland's University Hospital (Umeå), Lund Children's Hospital, Lomma Primary Health Care Centre, and the BAMSE team and Centrallasarettet in Växjö for their great collaboration during our visits for clinical examinations.

Financial support was provided by the Swedish Research Council (grant number 2011-3060) and through the Swedish Initiative for research on Microdata in the Social And Medical Sciences (SIMSAM) framework grant number 340-2013-5867, grants provided by the Stockholm 
County Council (ALF project no 520344 and 530422), the Strategic Research Program in Epidemiology at Karolinska Institutet and the Swedish Asthma and Allergy Association's Research Foundation (grant numbers 2010048-K, 2011051-K, 2012056-K, 2013049-K), Stiftelsen Frimurare Barnahuset Stockholm and Sällskapet Barnavård (grant number 2328013).

\section{List of Abbreviations}

CATSS: Child and Adolescent Twin Study in Sweden

CBC: complete cell count

FeNO: fractional exhaled NO

STOPPA: Swedish Twin study On Prediction and Prevention of Asthma

ACC: Asthma Concordant

ADC: Asthma Discordant

HCC: Healthy Concordant

\section{References}

Almqvist, C., Adami, H. O., Franks, P. W., Groop, L., Ingelsson, E., Kere, J., ... Pedersen, N. L. (2011). LifeGene -- A large prospective population-based study of global relevance. European Journal of Epidemiology, 26, 6777.

Anckarsater, H., Lundstrom, S., Kollberg, L., Kerekes, N., Palm, C., Carlstrom, E., ... Lichtenstein, P. (2011). The child and adolescent twin study in Sweden (CATSS). Twin Research and Human Genetics, 14, 495-508.

Asher, M. I., Keil, U., Anderson, H. R., Beasley, R., Crane, J., Martinez, F., ... Williams, H. C. (1995). International study of asthma and allergies in childhood (ISAAC): Rationale and methods. European Respiratory Journal, 8, 483491.

Asher, M. I., Montefort, S., Bjorksten, B., Lai, C. K., Strachan, D. P., Weiland, S. K., ... Williams, H. (2006). Worldwide time trends in the prevalence of symptoms of asthma, allergic rhinoconjunctivitis, and eczema in childhood: ISAAC phases one and three repeat multicountry cross-sectional surveys. Lancet, 368, 733-743.

Barker, D. J. (1995). Fetal origins of coronary heart disease. BMJ, 311, 171-174.

Blaser, M. J., Chen, Y., \& Reibman, J. (2008). Does Helicobacter pylori protect against asthma and allergy? Gut, 57, 561-567.

Braun-Fahrlander, C., Gassner, M., Grize, L., Neu, U., Sennhauser, F. H., Varonier, H. S., ... Wuthrich, B. (1999). Prevalence of hay fever and allergic sensitization in farmers' children and their peers living in the same rural community. SCARPOL team. Swiss Study on childhood allergy and respiratory symptoms with respect to air pollution. Clinical \& Experimental Allergy, 29, 28-34.

Cohen, J., Postma, D. S., Douma, W. R., Vonk, J. M., De Boer, A. H., \& ten Hacken, N. H. (2011). Particle size matters: Diagnostics and treatment of small airways involvement in asthma. European Respiratory Journal, 37, 532-540.

Custovic, A., \& Simpson, A. (2004). Environmental allergen exposure, sensitisation and asthma: From whole populations to individuals at risk. Thorax, 59, 825-827.
Danell, C. S., Bergstrom, A., Wahlgren, C. F., Hallner, E., Bohme, M., \& Kull, I. (2013). Parents and school children reported symptoms and treatment of allergic disease differently. Journal of Clinical Epidemiology, 66, 783-789. doi:10.1016/j.jclinepi.2013.02.006.

Drever, N., Saade, G. R., \& Bytautiene, E. (2010). Fetal programming: Early-life modulations that affect adult outcomes. Current Allergy and Asthma Reports, 10, 453459.

Dweik, R. A., Boggs, P. B., Erzurum, S. C., Irvin, C. G., Leigh, M. W., Lundberg, J. O., ... Taylor, D. R. (2011). An official ATS clinical practice guideline: Interpretation of exhaled nitric oxide levels (FENO) for clinical applications. American Journal of Respiratory and Critical Care Medicine, 184, 602-615.

Ekström, S., Magnusson, J., Kull, I., Lind, T., Almqvist, C., Melén, E., \& Bergström, A. (2015). Maternal body mass index in early pregnancy and offspring asthma, rhinitis and eczema up to 16 years of age. Clin. Exp. Allergy, 45(1), 283 91.

Fang, F., Hoglund, C. O., Arck, P., Lundholm, C., Langstrom, N., Lichtenstein, P., ... Almqvist, C. (2011). Maternal bereavement and childhood asthma-analyses in two large samples of Swedish children. PLoS One, 6, e27202.

Hollingsworth, J. W., Maruoka, S., Boon, K., Garantziotis, S., Li, Z., Tomfohr, J., ... Schwartz, D. A. (2008). In utero supplementation with methyl donors enhances allergic airway disease in mice. Journal of Clinical Investigation, 118, 3462-3469.

Lau, S., Nickel, R., Niggemann, B., Gruber, C., Sommerfeld, C., Illi, S., ... von Mutius, E. (2002). The development of childhood asthma: Lessons from the German Multicentre Allergy Study (MAS). Paediatric Respiratory Reviews, 3, 265-272.

Lichtenstein, P., De Faire, U., Floderus, B., Svartengren, M., Svedberg, P., \& Pedersen, N. L. (2002). The Swedish Twin Registry: A unique resource for clinical, epidemiological and genetic studies. Journal of Internal Medicine, 252, 184205.

Lichtenstein, P., \& Svartengren, M. (1997). Genes, environments, and sex: Factors of importance in atopic diseases in 7-9-year-old Swedish twins. Allergy, 52, 1079-1086.

Liu, A. H., Zeiger, R. S., Sorkness, C. A., Ostrom, N. K., Chipps, B. E., Rosa, K., . . McDonald, J. (2010). The Childhood Asthma Control Test: Retrospective determination and clinical validation of a cut point to identify children with very poorly controlled asthma. Journal of Allergy and Clinical Immunology, 126, 267-273.

Liu, J., Ballaney, M., Al-alem, U., Quan, C., Jin, X., Perera, F., ... Miller, R. L. (2008). Combined inhaled diesel exhaust particles and allergen exposure alter methylation of Thelper genes and IgE production in vivo. Toxicological Sciences, 102, 76-81.

Los, H., Postmus, P. E., \& Boomsma, D. I. (2001). Asthma genetics and intermediate phenotypes: A review from twin studies. Twin Research, 4, 81-93.

Lund, M. B., Kongerud, J., Nystad, W., Boe, J., \& Harris, J. R. (2007). Genetic and environmental effects on exhaled 
nitric oxide and airway responsiveness in a populationbased sample of twins. European Respiratory Journal, 29, 292-298.

Lundholm, C., Ortqvist, A. K., Lichtenstein, P., Cnattingius, S., \& Almqvist, C. (2010). Impaired fetal growth decreases the risk of childhood atopic eczema: A Swedish twin study. Clinical \& Experimental Allergy, 40, 1044-1053.

Magnusson, P. K., Almqvist, C., Rahman, I., Ganna, A., Viktorin, A., Walum, H., ... Lichtenstein, P. (2013). The Swedish Twin Registry: Establishment of a biobank and other recent developments. Twin Research and Human Genetics, 16, 317-329.

Martino, D., Loke, Y. J., Gordon, L., Ollikainen, M., Cruickshank, M. N., Saffery, R., \& Craig, J. M. (2013). Longitudinal, genome-scale analysis of DNA methylation in twins from birth to 18 months of age reveals rapid epigenetic change in early life and pair-specific effects of discordance. Genome Biology, 14, R42.

Matricardi, P. M. (2002). Probiotics against allergy: Data, doubts, and perspectives. Allergy, 57, 185-187.

Mihrshahi, S., Peat, J. K., Webb, K., Tovey, E. R., Marks, G. B., Mellis, C. M., \& Leeder, S. R. (2001). The childhood asthma prevention study (CAPS): Design and research protocol of a randomized trial for the primary prevention of asthma. Controlled Clinical Trials, 22, 333-354.

Miller, M. R., Hankinson, J., Brusasco, V., Burgos, F., Casaburi, R., Coates, A., ... Wanger, J. (2005). Standardisation of spirometry. European Respiratory Journal, 26, 319-338.

Nathan, R. A., Sorkness, C. A., Kosinski, M., Schatz, M., Li, J. T., Marcus, P., ... Pendergraft, T. B. (2004). Development of the asthma control test: A survey for assessing asthma control. Journal of Allergy and Clinical Immunology, 113, 59-65.

Noverr, M. C., \& Huffnagle, G. B. (2005). The 'microflora hypothesis' of allergic diseases. Clinical \& Experimental Allergy, 35, 1511-1520.

Ortqvist, A. K., Lundholm, C., Carlstrom, E., Lichtenstein, P., Cnattingius, S., \& Almqvist, C. (2009). Familial factors do not confound the association between birth weight and childhood asthma. Pediatrics, 124, e737-743.

Ortqvist, A. K., Lundholm, C., Wettermark, B., Ludvigsson, J. F., Ye, W., \& Almqvist, C. (2013). Validation of asthma and eczema in population-based Swedish drug and patient registers. Pharmacoepidemiology and Drug Safety, 22, 850860

Osika, W., Friberg, P., \& Wahrborg, P. (2007). A new short self-rating questionnaire to assess stress in children. International Journal of Behavioral Medicine, 14, 108-117.
Petersen, A. C., Crockett, L., Richards, M., \& Boxer, A. (1988). A self-report measure of pubertal status: Reliability, validity, and initial norms. Journal of Youth and Adolescence, 17, 117133.

Snethen, J. A., Hewitt, J. B., \& Goretzke, M. (2007). Childhood obesity: The infancy connection. Journal of Obstetric, Gynecologic, \& Neonatal Nursing, 36, 501-510.

Stenius, F., Borres, M., Bottai, M., Lilja, G., Lindblad, F., Pershagen, G., ... Alm, J. (2011). Salivary cortisol levels and allergy in children: The ALADDIN birth cohort. Journal of Allergy and Clinical Immunology, 128, 13351339.

Stettler, N., Zemel, B. S., Kumanyika, S., \& Stallings, V. A. (2002). Infant weight gain and childhood overweight status in a multicenter, cohort study. Pediatrics, 109, 194199.

Strachan, D. P. (1989). Hay fever, hygiene, and household size. BMJ, 299, 1259-1260.

Vink, N. M., Boezen, H. M., Postma, D. S., \& Rosmalen, J. G. (2013). Basal or stress-induced cortisol and asthma development: The TRAILS study. European Respiratory Journal, 41, 846-852.

Wickman, M., Kull, I., Pershagen, G., \& Nordvall, S. L. (2002). The BAMSE project: Presentation of a prospective longitudinal birth cohort study. Pediatric Allergy and Immunology, 13, 11-13.

Wijga, A., Smit, H. A., Brunekreef, B., Gerritsen, J., Kerkhof, M., Koopman, L. P., \& Neijens, H. J. (2001). Are children at high familial risk of developing allergy born into a low risk environment? The PIAMA Birth Cohort Study. Prevention and incidence of asthma and mite allergy. Clinical \& Experimental Allergy, 31, 576-581.

Wong, C. C., Caspi, A., Williams, B., Craig, I. W., Houts, R., Ambler, A., ... Mill, J. (2010). A longitudinal study of epigenetic variation in twins. Epigenetics, 5, 516526.

Wu, T., Boezen, H. M., Postma, D. S., Los, H., Postmus, P. E., Snieder, H., \& Boomsma, D. I. (2010). Genetic and environmental influences on objective intermediate asthma phenotypes in Dutch twins. European Respiratory Journal, 36, 261-268.

Zhang, D., Li, S., Tan, Q., \& Pang, Z. (2012). Twin-based DNA methylation analysis takes the center stage of studies of human complex diseases. Journal of Genetics and Genomics, $39,581-586$. 